Research Article

\title{
Compressive Strength Evaluation of Fiber-Reinforced High-Strength Self-Compacting Concrete with Artificial Intelligence
}

\author{
Tu T. Nguyen $\mathbb{D}^{1},{ }^{1}$ Hoa Pham Duy, ${ }^{2}$ Tung Pham Thanh, ${ }^{3}$ and Hoang Hiep Vu ${ }^{1}$ \\ ${ }^{1}$ Faculty of Civil Engineering, Hanoi Architectural University, Hanoi, Vietnam \\ ${ }^{2}$ Faculty of Bridge and Roads, National University of Civil Engineering, Hanoi, Vietnam \\ ${ }^{3}$ Faculty of Building and Industrial Construction, National University of Civil Engineering, Hanoi, Vietnam
}

Correspondence should be addressed to Tu T. Nguyen; tunt@hau.edu.vn

Received 6 December 2019; Revised 24 June 2020; Accepted 9 October 2020; Published 22 October 2020

Academic Editor: Weerachart Tangchirapat

Copyright $\odot 2020$ Tu T. Nguyen et al. This is an open access article distributed under the Creative Commons Attribution License, which permits unrestricted use, distribution, and reproduction in any medium, provided the original work is properly cited.

This paper describes the application of two artificial intelligence- (AI-) based methods to predict the 28-day compressive strength of fiber-reinforced high-strength self-compacting concrete (FRHSSCC) from its ingredients. A series of 131 data samples collected from various published literature sources were used for training, validation, and testing models. Various AI models were developed with different training algorithms and a number of nodes in the hidden layer to obtain the optimal model for the FRHSSCC data. It is shown that the performances of the artificial neural network (ANN) were better than that of the adaptive neurofuzzy inference system (ANFIS) model. Specifically, the overall coefficient of determination $\left(R^{2}\right)$ of the ANN and ANFIS models was 0.9742 and 0.9584 , respectively. The sensitivity analysis was also conducted with the ANN model to investigate the effects of input parameters on the output. The results from the sensitivity analysis revealed that the compressive strength of FRHSSCC at 28 days was more sensitive with the changes of water by cement ratio (WCR) parameter and insensitive with varying amounts of fiber (VOF). Finally, it can be concluded that the application of artificial intelligence shows the great potential in the prediction of compressive strength of FRHSSCC.

\section{Introduction}

Concrete is among the most widely used materials in civil buildings and other structures around the world. Conventional concrete is a mixture of water, cement, and aggregates [1]. Due to some limitations such as low strength/weight ratio, the conventional concrete is often suitable for low-rise civil buildings and typical span structures. Efforts of expanding the applications of concrete in the construction industry lead to the arrival of high-performance concrete (HPC). Unlike the traditional concrete, HPC is designed to achieve many exceptional properties such as more workability in the fresh state and high strength and durability in the hardened state [2].

Among many types of concrete in the HPC family, highstrength self-compacting concrete (HSSCC) is broadly utilized in the construction projects [3, 4]. HSSCC is often made by adding mineral admixtures (fly ash and blast furnace slag) and chemical materials (superplasticizer) to the conventional concrete mixture. Along with the development in the field of concrete technology, a new class of concrete, called fiber-reinforced high-strength self-compacting concrete, is developed. FRHSSCC is the combination of HSSCC with various kinds of fiber. The new construction material benefits many superior properties of HSSCC such as highly flowable, nonsegregating, as well as the ductility improvement from added fibers $[5,6]$.

As a crucial hardened property of FRHSSCC, the compressive strength and other material properties can be evaluated using the experimental tests. The method, however, not only is time-consuming to conduct but also requires intensive testing facilities and skilled personnel 
[7-10]. To overcome those obstacles, an alternative method to estimate the compressive strength of FRHSSCC using AI can be applied. The AI-based method used published experimental mixes from previous studies to develop the nonlinear implicit relationship between various input parameters with the compressive strength of FRHSSCC.

Within the various AI-based techniques, artificial neural networks and adaptive neurofuzzy inference system are the most commonly used methods for evaluating the hardened properties of the concrete material. For example, Ince employed the ANN model to predict the fracture parameters of concrete [11]. The ANN method was also applied for the prediction of compressive strength for different types of concrete such as concrete containing construction and demolition waste [12], lightweight concrete [13], geopolymer concrete [14], environment-friendly concrete [15], high-strength concrete [16], no-slump concrete [17], and HPC with the specific admixtures [18].

Not only the ANN model but also the ANFIS model was utilized by many researchers. For instance, Khademi et al. estimated the 28-day compressive strength of conventional concrete using ANN and ANFIS models [19]. Nguyen and Dinh applied the ANFIS method to estimate the 28-day compressive strength of concrete [20]. The application of ANFIS for the prediction of dry-cast concrete strength was found in a study by Sobhani and Najimi [21] as well. Besides the applications for prediction of concrete properties, the AI-based models have been successfully applied in a variety of fields, including air quality forecasting [22], bridge desk rating [23], and wastewater treatment plant optimization [24].

In a recent study, Armaghani et al. [25] applied an ANN method to predict the shear strength of the concrete beam. The conclusion from the study revealed that the shear strength of a reinforced concrete beam with and without stirrups could be successfully estimated. In another study by Sarir et al. [26], different AI-based models, including GEP tree-based, neuroswarm, and whale optimization, were used to evaluate the bearing capacity of concrete-filled steel tube columns. More recently, a modified ANFIS model was used to predict the uniaxial compressive strength of the rock in a study by Jing et al. [27].

More and more experimental data are available along with advanced AI applications recently, which enable developing a new technique to evaluate the compressive strength of FRHSSCC. To the best of the authors' knowledge, there has been no available research to apply the AI-based model for predicting the compressive strength of FRHSSCC. Accordingly, in this study, a series of 131 FRHSSCC experimental mix samples with the compressive strength up to $162 \mathrm{MPa}$ (the percentage in the volume of the fiber in the mixes is up to four percent) were carefully selected. A trialand-error procedure was utilized for this dataset to obtain the best AI model with the highest coefficient of determination $\left(R^{2}\right)$ and the lowest root mean squared error (RMSE). Additionally, the sensitivity analysis was performed for the selected model to evaluate the effects of input parameters on the output.
1.1. Data Preparation. The original experimental data were obtained from the previous study by Salari et al. [5]. Nine FRHSSCC properties, namely, fiber type (FBT), percentage in the volume of fiber (VOF), cement (CEM), water (WTR), mineral powder (MPR), water by cement ratio (WCR), fine aggregate (FIA), coarse Aggregate (COA), and the 28-day compressive strength (F28), were used. Because of conflicting information in the original data, the data need to be refined to attain reliable inputs. The following criteria were applied in the refinement procedures: (i) only mixtures with the amount of fiber in percentage were selected, (ii) mixtures with the amount of water not in $\mathrm{kg} / \mathrm{m}^{3}$ unit were excluded, and (iii)mixtures with no information about compressive strength were not selected. In the final refinement stage, the chemical admixture column was removed from the database due to the inconsistent units between mixtures.

The refined database was a matrix with 131 rows and 9 columns. Each row in the database matrix contained nine important properties of FRHSSCC in which data from column 1 to column 8 were used as the inputs and compressive strength data in column 9 were the output. Seven types of fiber were utilized in the experimental dataset: (1) steel, (2) polyphenylene sulfide, (3) glass fiber-reinforced polymer, (4)wave steel fiber, (5) hook-ended steel fiber, (6) flat steel fiber, and (7) the combination of steel and one of the fibers. The ranges of the input and output parameters are listed in Table 1.

The classifications of the 28-day compressive strength of FRHSSCC in each specific interval are presented in Table 2. The distributions of the four considered input parameters in this study are shown in Figure 1.

\section{Methods}

2.1. Model Architecture. Two supervised learning AI-based models were employed in this study to predict the 28-day compressive strength of FRHSSCC. In the first approach, the artificial neural networks model was used. Unlike the conventional approaches, which chiefly rely on pure mathematical models, the Artificial Neural Networks model simulates the function of the biological nervous systems in the human brain to solve the problems. Basically, an ANN model contains a collection of simple processing units (or neurons) working independently and fully connecting to the others as a network. A neuron receives input values from neurons on the previous layers, computes an output, and sends the results to all connected neurons on the next layer.

Figure 2 depicts a schematic for a neuron. It consists of five main parts: (i) inputs or information that enters the neuron, (ii) weight or effect of an input set, (iii) sum function or a function that calculates net input that comes to a neuron, (iv) activation function or a function that processes the net input and determines the neuron output, and (v) outputs.

In the second approach, the ANFIS model was utilized. ANFIS is a combination of two machine learning methods (fuzzy logic and neural network). In an ANFIS model, the parameters of a fuzzy inference system (FIS) were tuned by 
TABLE 1: Characteristics of input and output.

\begin{tabular}{|c|c|c|c|c|c|c|c|c|c|}
\hline No. & FBT & VOF (\%) & CEM $\left(\mathrm{kg} / \mathrm{m}^{3}\right)$ & WTR $\left(\mathrm{kg} / \mathrm{m}^{3}\right)$ & MPR $\left(\mathrm{kg} / \mathrm{m}^{3}\right)$ & WCR & FIA $\left(\mathrm{kg} / \mathrm{m}^{3}\right)$ & $\operatorname{COA}\left(\mathrm{kg} / \mathrm{m}^{3}\right)$ & F28 (MPa) \\
\hline 1 & 2 & 0.4 & 413 & 162 & 288.9 & 0.39 & 826 & 772 & 67.4 \\
\hline 2 & 1 & 0.5 & 425 & 192 & 200 & 0.45 & 740 & 814 & 57.1 \\
\hline 3 & 1 & 1 & 425 & 192 & 200 & 0.45 & 740 & 814 & 53.6 \\
\hline - & & - & - & - & - & - & - & - & - \\
\hline - & & - & - & - & - & - & - & - & - \\
\hline 129 & 6 & 3.5 & 473 & 162 & 83.1 & 0.34 & 702 & 1042 & 81.6 \\
\hline 130 & 6 & 4 & 473 & 162 & 83.1 & 0.34 & 702 & 1042 & 80.2 \\
\hline 131 & 7 & 0.75 & 640 & 181 & 224 & 0.28 & 887 & 798 & 54 \\
\hline Min. & 1 & 0 & 300 & 138 & 70 & 0.22 & 451 & 0 & 50 \\
\hline Max. & 7 & 4 & 900 & 243 & 691 & 0.58 & 1233 & 1042 & 162 \\
\hline
\end{tabular}

TABLE 2: Number of samples in each specific range of 28-day compressive strength.

\begin{tabular}{lcc}
\hline No. & 28-day compressive strength $(\mathrm{MPa})$ & Number of samples \\
\hline 1 & $<75$ & 46 \\
2 & $75-100$ & 33 \\
3 & $100-125$ & 35 \\
4 & $>125$ & 17 \\
Total & & 131 \\
\hline
\end{tabular}

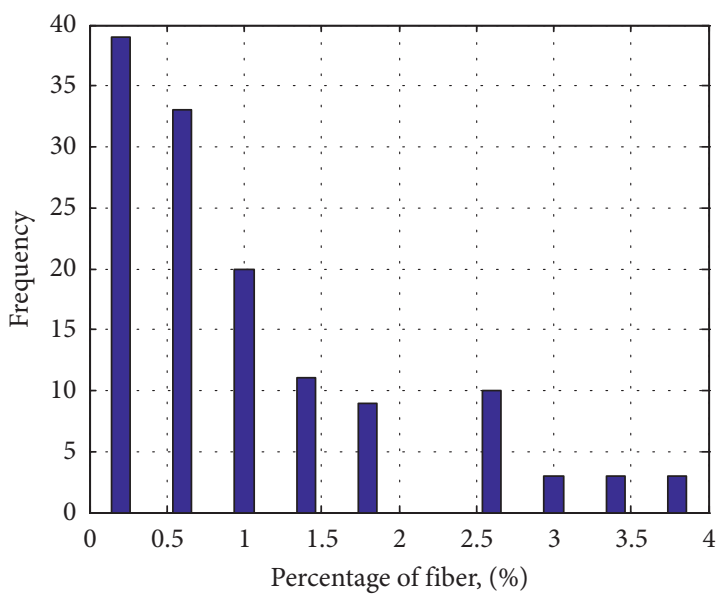

(a)

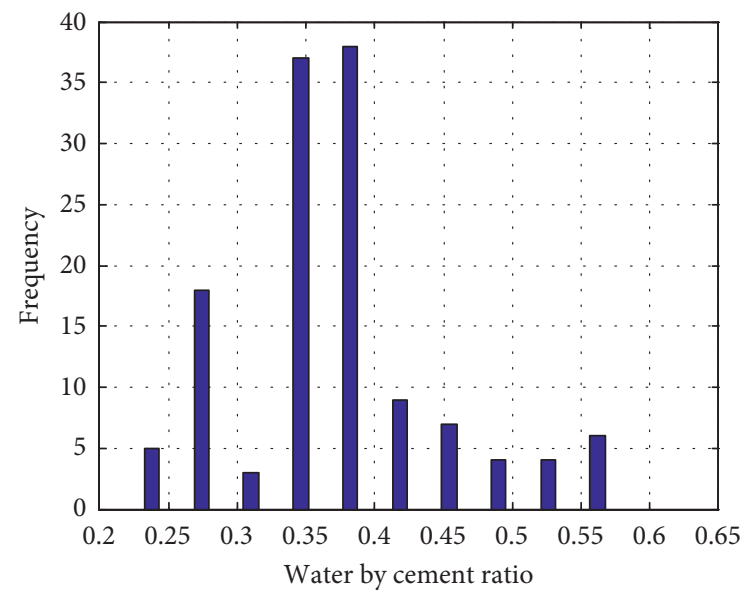

(c)

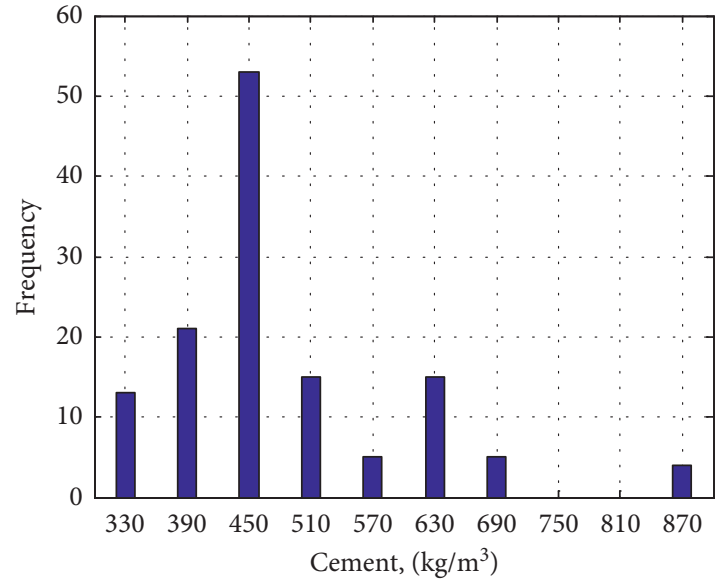

(b)

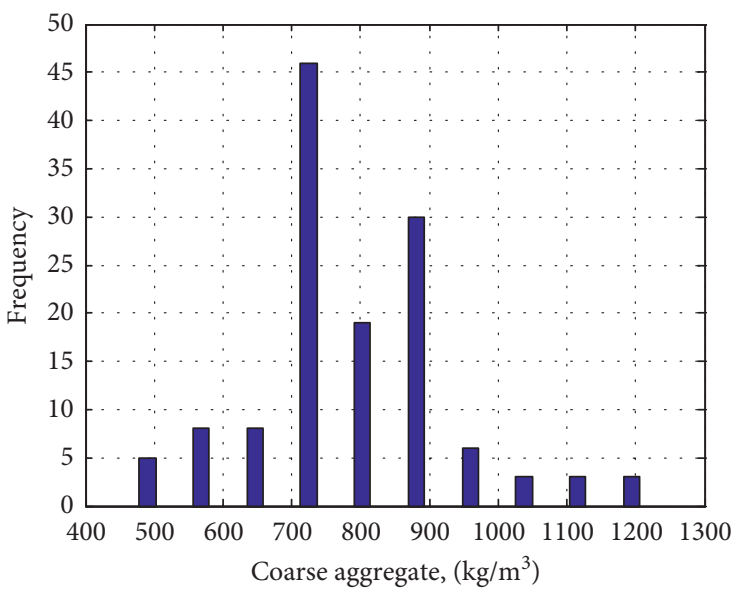

(d)

FIGURE 1: Distribution of four selected input parameters. 


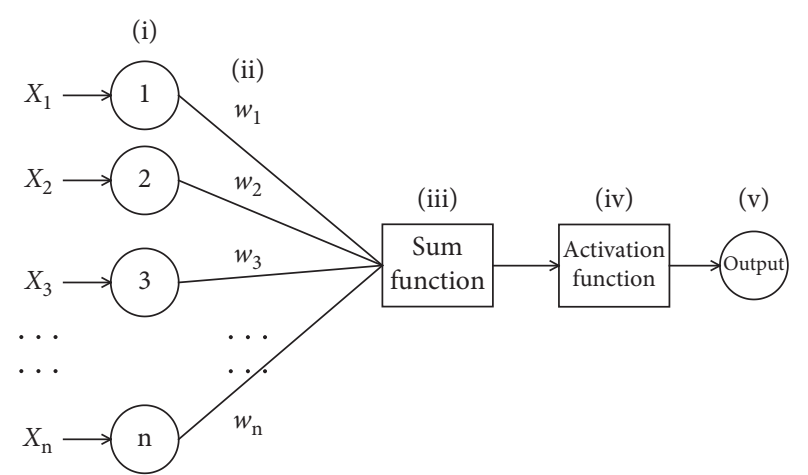

Figure 2: A simple neuron architecture.

using the neural network learning method. The basic ANFIS architecture with two input variables is shown in Figure 3. In this architecture, two fuzzy IF-THEN rules based on a firstorder Sugeno model are presented.

Rule 1: if $x$ is $A_{1}$ and $y$ is $B_{1}$, then $f_{1}=p_{1} x+q_{1} y+r_{1}$

Rule 2: if $x$ is $A_{2}$ and $y$ is $B_{2}$, then $f_{2}=p_{2} x+q_{2} y+r_{2}$ where $x$ and $y$ are the inputs; $A_{i}$ and $B_{i}$ are the fuzzy sets; $f_{i}$ are the outputs within the fuzzy region specified by the fuzzy rule; $p_{i}, q_{i}$, and $r_{i}$ are the design parameters that are determined during the training process.

2.2. Performance Criteria. The performances of the ANN and ANFIS models were evaluated based on four indicators: coefficient of determination $\left(R^{2}\right)$, mean squared error

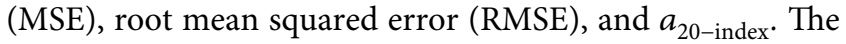
coefficient of determination measures the correlation between input and output variables using the following equation:

$$
R^{2}=1-\frac{\sum_{i=1}^{n}\left(y_{i}-\widehat{y}_{i}\right)^{2}}{\sum_{i=1}^{n}\left(y_{i}-\bar{y}\right)^{2}},
$$

where $y_{i}$ is the $i^{\text {th }}$ actual output, $\bar{y}$ is the mean of the actual outputs, $\widehat{y}_{i}$ are the $i^{\text {th }}$ predicted outputs, and $n$ is the total number of samples. MSE is the average squared difference between predicted outputs and actual outputs. MSE can be calculated using the following equation:

$$
\text { MSE }=\frac{1}{n} \sum_{i=1}^{n}\left(y_{i}-\widehat{y}_{i}\right)^{2} .
$$

Root mean squared error is the square root of mean squared error and can be calculated by the following equation:

$$
\mathrm{RMSE}=\sqrt{\frac{1}{n} \sum_{i=1}^{n}\left(y_{i}-\hat{y}_{i}\right)^{2}} .
$$

Another index, called $a_{20 \text {-index }}$, was also used in this study to evaluate the performance of the models. This index presents the number of samples with the difference between the predicted value and experimental value within \pm 20

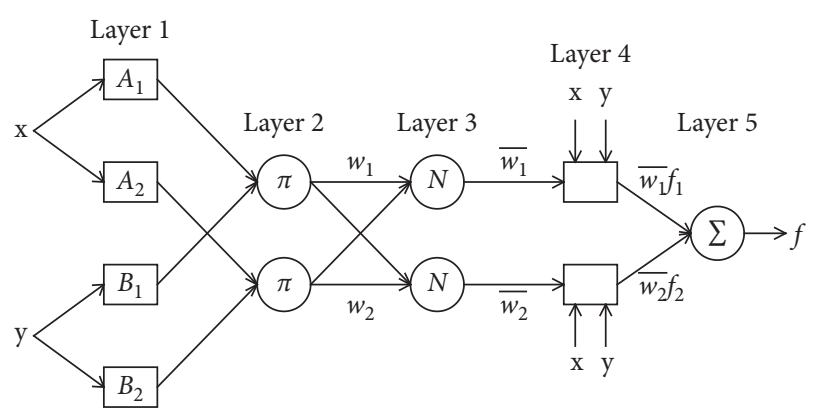

FIgure 3: Structure of the ANFIS model.

percent $[25,28] . a_{20 \text {-index }}$ can be calculated by the following equation:

$$
a_{20-\text { index }}=\frac{m_{20}}{M}
$$

where $m_{20}$ is the number of samples with the ratio of experimental value over predicted value falling from 0.8 to 1.2 and $M$ is the number of samples in the dataset.

2.3. Model Development. Eight FRHSSCC properties, fiber type, percentage in the volume of fiber, cement, water, and mineral powder, water by cement ratio, fine aggregate, and coarse aggregate, were selected as the input parameters of the ANN model, and the 28-day compressive strength of FRHSSCC (F28) was assigned as the output. The dataset was divided randomly into three subsets, in which $70 \%$ (i.e., 91 data samples) of the entire dataset was employed for training model, $15 \%$ (i.e., 20 data samples) for validation, and the remaining 15\% (i.e., 20 data samples) was utilized for testing the prediction accuracy of the ANN model.

Neural Networks learn to map between input and output through a common learning algorithm called feed-forward backpropagation. It works by using the errors presented in the network output to adjust the weights in each layer. The error backpropagation algorithm consists of two different processes: one is a feed-forward process, and the other is a backpropagation process. In the feed-forward process, the inputs are used to obtain the outputs with some network errors. The errors are then passed backwards to the input layers through the backpropagation process, and the weights are adjusted during this process to minimize the network errors to an acceptable level.

In order to find the best learning algorithm that works for the available data, eight ANN models were developed and tested using different learning algorithms. The performances of the models were evaluated based on the values of four parameters, namely, training performance (TRN), testing performance (TES), validation performance (VAL), and the number of epochs (EPC). To obtain a reliable result, five trials were performed for each model. The best values of the performance results from those models are listed in Table 3. Apparently, the Levenberg-Marquardt (LM) algorithm (Trainlm) produces the best performance on training, testing, and validation with a low number of epochs. The results were in line with previous studies [29]. For this reason, the LM algorithm was selected for the proposed ANN model. 
TABle 3: Performance of the ANN model with different learning algorithms.

\begin{tabular}{|c|c|c|c|c|c|c|}
\hline No. & Algorithm & Details & TRN & TES & VAL & EPC \\
\hline 1 & Trainrp & Resilient backpropagation & 70.8 & 42.5 & 25.3 & 6 \\
\hline 2 & Trainlm & Levenberg-Marquardt backpropagation & 8.29 & 31.6 & 21.2 & 6 \\
\hline 3 & Traincgp & Conjugate gradient backpropagation with Polack-Ribiere updates & 131 & 43.9 & 105 & 6 \\
\hline 4 & Traincgb & Conjugate gradient backpropagation with Power-Beale restarts & 71.9 & 38.2 & 76.9 & 6 \\
\hline 5 & Trainbfg & BFGS quasi-Newton backpropagation & 78.4 & 36.0 & 28.9 & 7 \\
\hline 6 & Trainoss & One-step secant backpropagation & 93.6 & 88.0 & 36.9 & 6 \\
\hline 7 & Traincgf & Conjugate gradient backpropagation with Fletcher-Reeves updates & 69.9 & 56.5 & 38.8 & 7 \\
\hline 8 & Traingda & Gradient descent with adaptive learning rate backpropagation & 192 & 128 & 113 & 20 \\
\hline
\end{tabular}

A sigmoid function was selected as an activation function in the proposed ANN model. The shape of the sigmoid function is depicted in Figure 4(a). Equation (5) expresses the mathematical form of the sigmoid function. This function transforms the values between 0 and 1 .

$$
y(x)=\frac{1}{1+e^{-x}} .
$$

According to a study by Nikbin et al. [29], a network with one hidden layer was adequate for solving most of the engineering problems. Thus, the ANN model with one hidden layer was chosen for this study. To determine the sufficient number of nodes in the hidden layer of the proposed ANN model, 20 ANN models were developed, with the number of nodes in the hidden layer varying from one node to 20 nodes. Each model was performed five times to obtain the average performance results. The performance of the ANN models was then evaluated based on the MSE values of the training, testing, and validation with the identical datasets. Figure 4(b) shows the average values of the performance results from these ANN models. It can be clearly observed that the ANN model with six neurons/ nodes yielded the best results. Therefore, the selected ANN model consisted of one hidden layer with six nodes. Detailed information on the selected ANN model is listed in Table 4.

The ANFIS model was also used in this study. The numbers of input and output parameters of the ANFIS model were similar to those of the ANN model. The dataset utilized for the ANFIS model was an identical dataset that was applied for the ANN model. However, the dataset was randomly divided into two subsets in which the training data subset contains about $80 \%$ of the entire data, that is, 105 data samples, and the testing data subset containing $20 \%$ of the entire data, that is, 26 data samples. Different type of membership functions was examined, and a "gauss2mf" function was selected for constructing the ANFIS structure. In the training process, the back-propaganda optimization method was applied with a zero value of error tolerance. The number of epochs/iterations for both training and testing stages was designated as 100 . The detailed structure of the ANFIS model is presented in Table 5.

\section{Results and Discussion}

There were two phases in this study. In the first phase, the performances of the ANN and ANFIS models were evaluated. All seven inputs were employed for the ANN and
ANFIS models to predict the 28-day compressive strength of FRHSSCC. The model with better performance was selected for use in the next phase of this study. In the second phase, the sensitivity analysis was conducted using the selected model. The main objective of the sensitivity analysis was to identify the significance of the inputs that influence the outcomes of the model.

3.1. Performance of the ANN Model. As mentioned above, seven FRHSSCC properties were used as the inputs for the ANN model. The output was the 28-day compressive strength of FRHSSCC. Several trials were performed to determine an optimal number of the hidden layers as well as the number of neurons inside the ANN structure. One hidden layer with six neurons was found to be adequate for this ANN model. The test results from the proposed ANN model in different stages are presented in Table 6 . The value of $R^{2}$ presents the ability of a model to predict the outputs based on the inputs. Values closer to 1 indicate a strong ability of the model in prediction. Performances of the ANN model for the training and validation stage are presented in Figure 5.

The ANN model performed well in training, validation, and testing with values of $R^{2}$ being $0.9755,0.9851$, and 0.9438 , respectively. Overall $R^{2}$ was 0.9742 , indicating a good prediction ability of the proposed ANN model. It is worth noting that the validation and testing datasets were unseen in the ANN model. However, $R^{2}$ produced by the ANN model in both validation and testing sets showed a minimal difference compared to $R^{2}$ calculated for the training dataset. That means the proposed ANN model is able to generalize and can be employed as a tool to predict the compressive strength of FRHSSCC.

Besides $R^{2}$, another indicator, RMSE, can also be employed to evaluate the performance of the ANN model. The RMSE values between predicted compressive strength of FRHSSCC and the experimental results are listed in Table 6. The smaller the RMSE value is, the stronger the linear relationship between variables is. As can be seen, the smallest RMSE value of $6.70 \mathrm{MPa}$ was recorded for the validation dataset. The value of RMSE was found to be higher for the new/unseen dataset, which was $8.71 \mathrm{MPa}$ for the testing dataset.

In order to have a clear overall view of the performance of the proposed ANN model, the predicted values of 28-day compressive strength and its experimental results for the entire specimens are plotted in Figure 6(a). In addition, the 


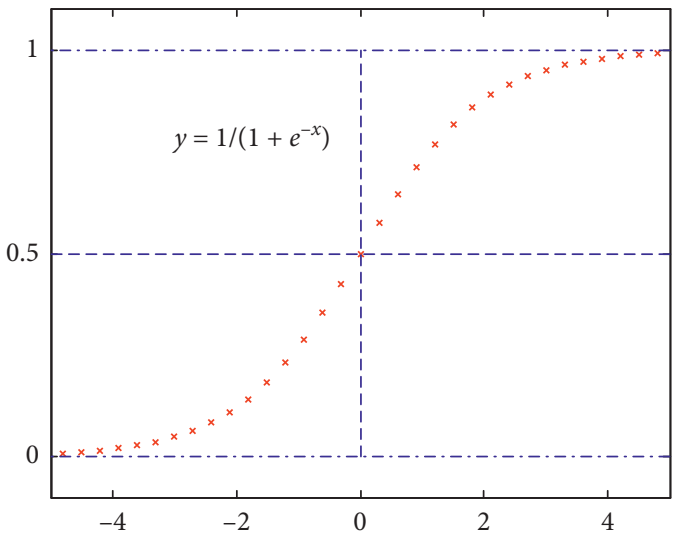

(a)

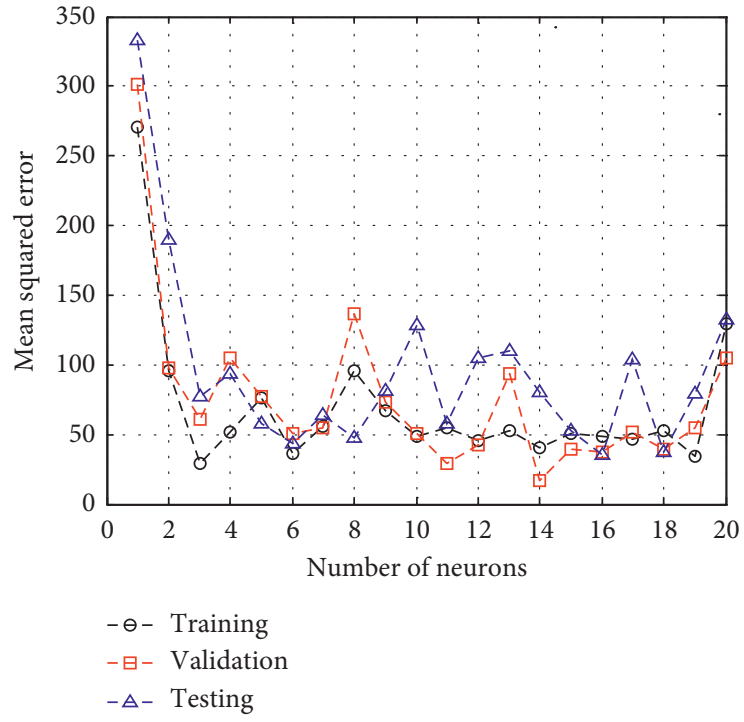

(b)

FIgURe 4: Activation function and model performance. (a) Sigmoid function. (b) Average results from 20 ANN models.

TABLE 4: Detailed information about the selected ANN model.

\begin{tabular}{lc}
\hline Parameter & Information \\
\hline $\begin{array}{l}\text { Number of neurons in the input layer } \\
\text { Number of neurons in the output layer }\end{array}$ & 8 \\
Training method & 1 \\
Training algorithm & Backpropagation \\
Levenberg-Marquardt & (Trainlm) \\
Activation function & Sigmoid \\
Number of hidden layers & 1 \\
Number of neurons in the hidden layer & 6 \\
\hline
\end{tabular}

TABLE 5: Detailed information about the selected ANFIS model.

\begin{tabular}{lc}
\hline Information & Value \\
\hline Number of nodes & 555 \\
Number of linear parameters & 2304 \\
Number of nonlinear parameters & 64 \\
Total number of parameters & 2368 \\
Number of training data pairs & 105 \\
Number of fuzzy rules & 256 \\
\hline
\end{tabular}

TABLE 6: Performance results of the ANN model.

\begin{tabular}{lcccc}
\hline & Training & Validation & Testing & Overall \\
\hline$R^{2}$ & 0.9755 & 0.9851 & 0.9438 & 0.9742 \\
MSE & 53.8 & 44.9 & 75.9 & 55.8 \\
RMSE & 7.33 & 6.70 & 8.71 & 7.47 \\
$a_{20}-$ index & 0.95 & 0.90 & 0.90 & 0.93 \\
Number of samples & 91 & 20 & 20 & 131 \\
\hline
\end{tabular}

absolute prediction errors in terms of percentage for all specimens/mixtures in the dataset are depicted in Figure 6(b).

As can be seen in Figure 6(a), the proposed ANN model estimated the 28-day compressive strength of FRHSSCC at a high level of accuracy. The absolute mean value of prediction errors was only about 5.76 percent, as presented in Figure 6(b). The results showed that the output values could be achieved from the input parameters. Several abnormal absolute errors were found in specimen numbers $29,54,84$, 89, and 126, as seen in Figure 6(b). The possible explanation for this might be due to the limited number of samples in the original dataset. As a result, the data were not sufficient to represent all aspects of the input variables. This issue may resolve when a dataset with a large number of samples was applied.

The linear regression plot was used in this study to present the results from the proposed ANN model. The plots for the performance of the proposed ANN model at different stages, namely, training, validation, testing, and overall, are shown, respectively, in Figures 7(a)-7(d). In these figures, the linear fitting line presents the relationship between the experimental results and the predicted values produced from the model. In addition, the " $x=y$ " line shows a perfect correlation between inputs and outputs.

3.2. Performance of the ANFIS Model. As previously discussed, the inputs and output for this ANFIS model were identified as used for the ANN model. The same dataset was employed for the ANFIS model; however, the dataset in this case was only divided into training and testing sets. Figure 8 shows the accumulated root mean squared error of the ANFIS model for both training and testing datasets. The errors reduced significantly in the first 50 epochs, and the minimum errors were obtained at the epoch of 100 . The detailed performance results of the ANFIS model are listed in Table 7.

As can be seen in Table 7, the proposed ANFIS model performed well for the training dataset with values of $R^{2}$ and 


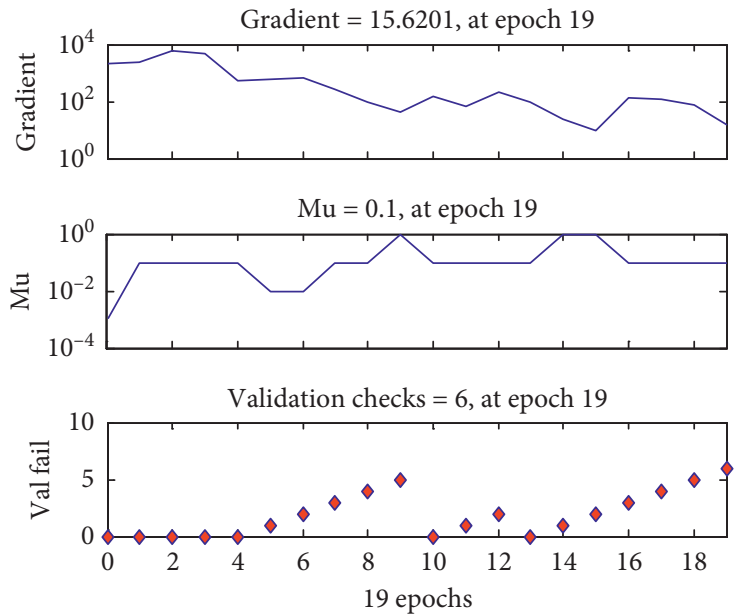

(a)

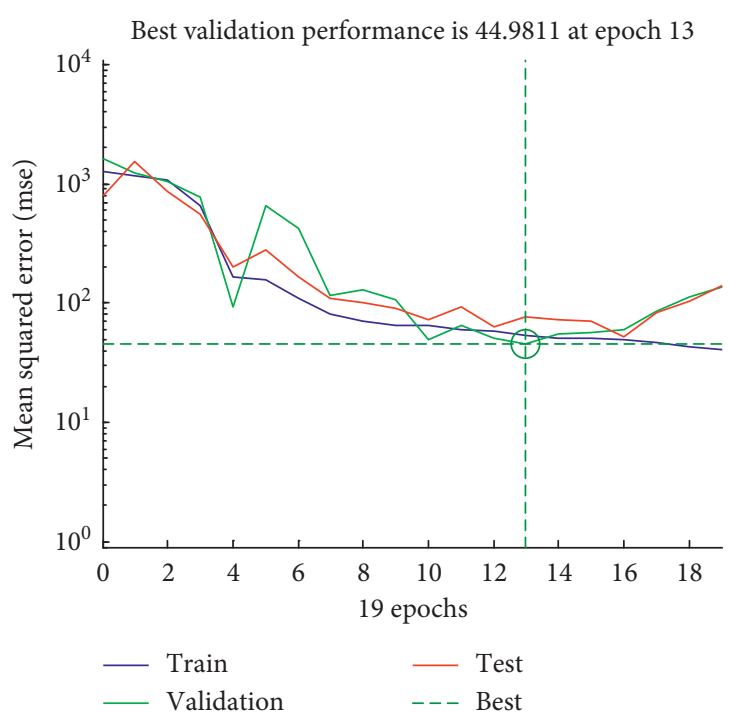

(b)

FIgURE 5: Information about the selected ANN model. (a) Training. (b) Validation.

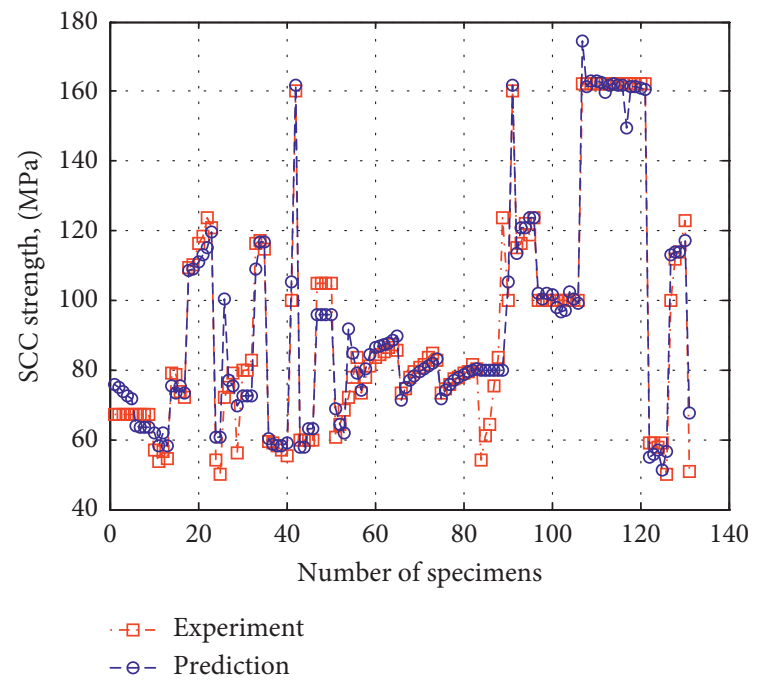

(a)

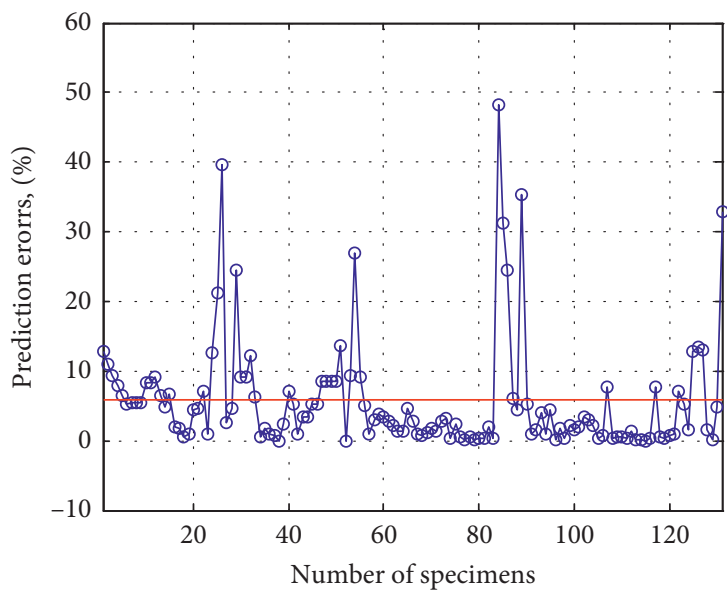

(b)

Figure 6: Performance of the ANN model. (a) Experiment versus prediction. (b) Absolute prediction errors.

RMSE being 0.9624 and 9.39, respectively. However, with the unseen dataset in the testing process, the value of $R^{2}$ was slightly smaller, which was 0.9518 . It is worth noting that the overall performance of this ANFIS model was reasonable, with a value of $R^{2}$ being 0.9584 . However, compared with the overall performance of the ANN model in the previous section, the ANFIS model showed a more deficient prediction capacity.

Figure 9(a) shows the plots of experimental compressive strength and the predicted values produced by the proposed ANFIS model. The absolute prediction errors in terms of percentage for the entire dataset can be seen in Figure 9(b). Although the average mean value of the prediction errors was at an acceptable level, at around seven percent, this model showed a low accuracy in prediction for unseen data. The majority of errors were found in the testing phase. Some predicted values experienced an error as large as 52 percent, as seen in Figure 9(b).

Figure 10(a) presents the linear regression plot of the results produced by the proposed ANFIS model for the training dataset. The linear regression plot for the overall performance can be seen in Figure 10(b).

The ANFIS model was also applied to examine the effects of the percentage of fiber and other input parameters on the 28-day compressive strength of FRHSSCC. The surface plots of the relationship between the VOF parameter and the four selected inputs are presented in Figure 11. 

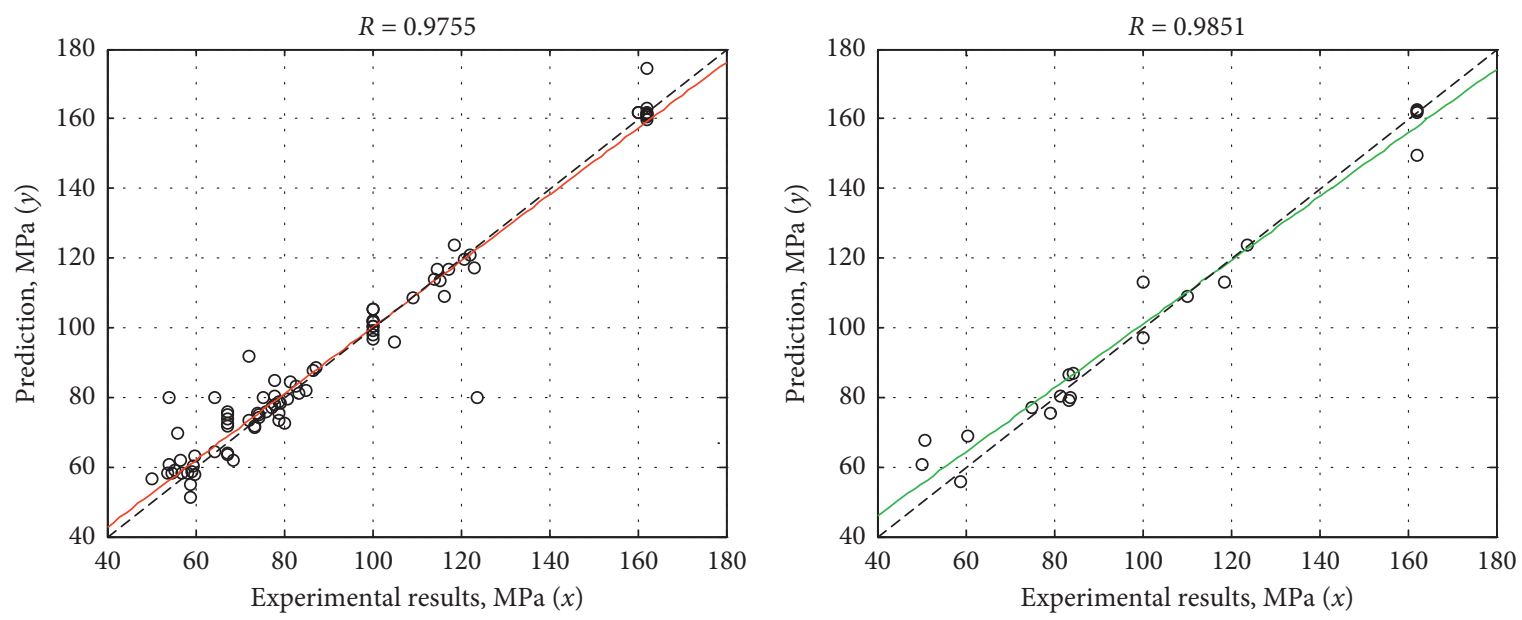

$\circ \quad$ Compressive strength
$-\quad$ Linear fitting
$---x=y$

(a)

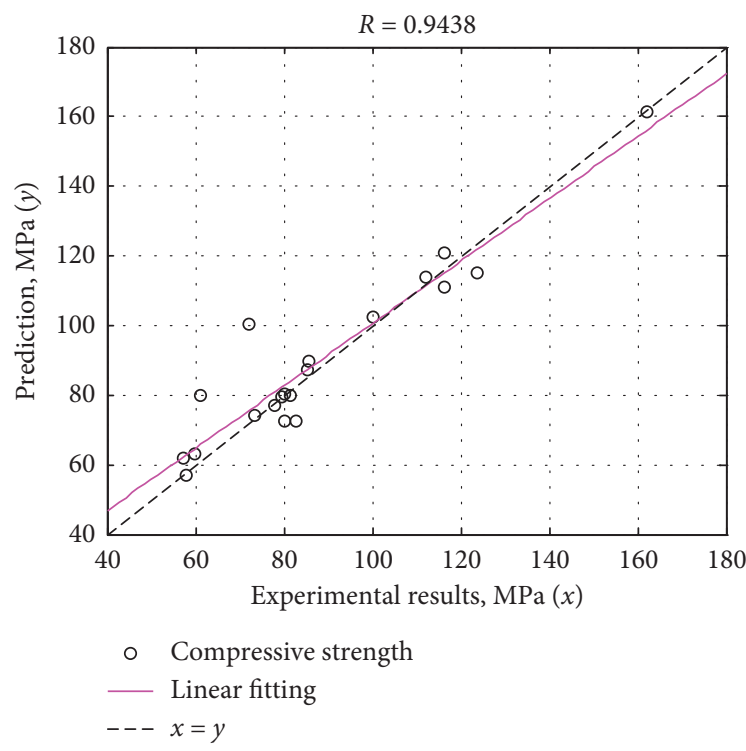

(c)
○ Compressive strength

_ Linear fitting

$--x=y$

(b)

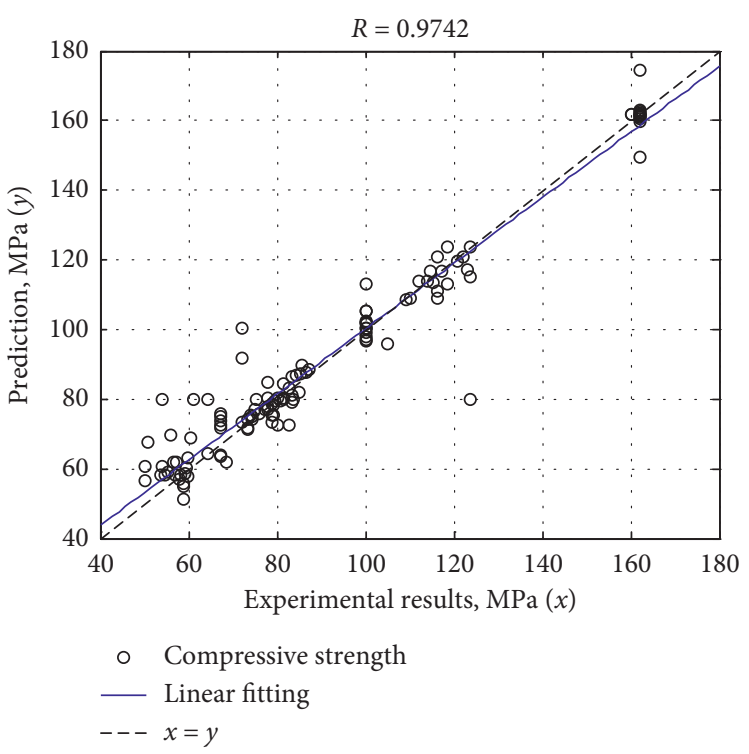

(d)

FIgURE 7: Linear regression plot of ANN performance. (a) Training. (b) Validation. (c) Testing. (d) Overall.

3.3. Model Selection. Results of the performance of the ANN and ANFIS models for the unseen dataset from the previous sections indicated that the ANN model had a higher prediction ability compared to that of the ANFIS model. Thus, within the context of this research, it was concluded that the performance of the ANN model in terms of $R^{2}$ for both testing data and overall was better than that of the ANFIS model. This conclusion is in line with a result of the previous study [18]. For that reason, the ANN model was selected to perform the sensitivity analysis in the subsequent sections.

3.4. Sensitivity Analysis. The sensitivity analysis was conducted for the selected ANN model to evaluate the influence of the inputs on the 28-day compressive strength of FRHSSCC. In order to perform the sensitivity analysis, each input parameter was divided into five groups, namely, Lowest (LO), Mid-Low (ML), Middle (MD), Mid-High $(\mathrm{MH})$, and Highest (HI). MD is the mean value of the LO and HI. ML and MH represent halfway from LO to MD and from MD to HI, respectively. Detailed values of these input parameters are listed in Table 8.

The sensitivity analysis was performed for each input parameter by changing its value from LO to $\mathrm{HI}$ while keeping the other inputs constant at the average values. The results of the sensitivity analysis for different input parameters are presented in Figure 12. It can be seen clearly that the 28-day FRHSSCC compressive strength was most sensitive to the water/cement ratio (WCR) and the amount of water content (WTR). This is reasonable since WCR and WTR are two main factors that govern the compressive 


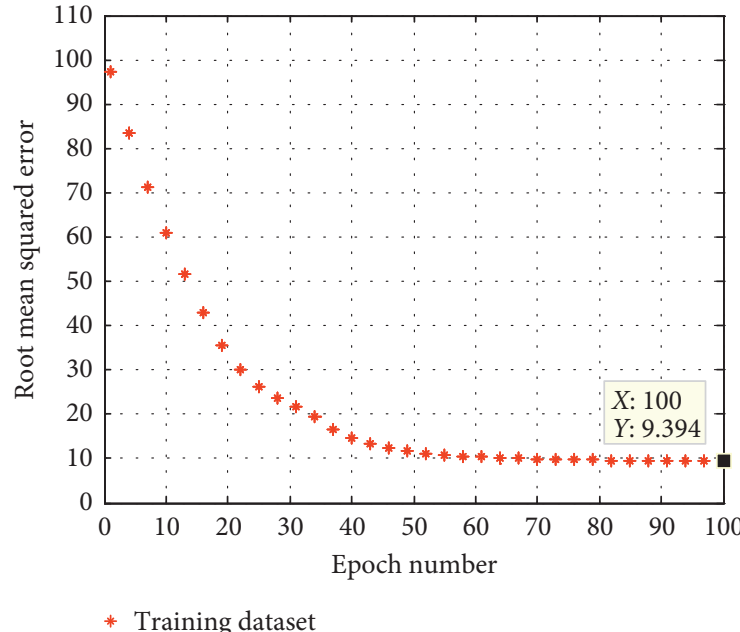

(a)

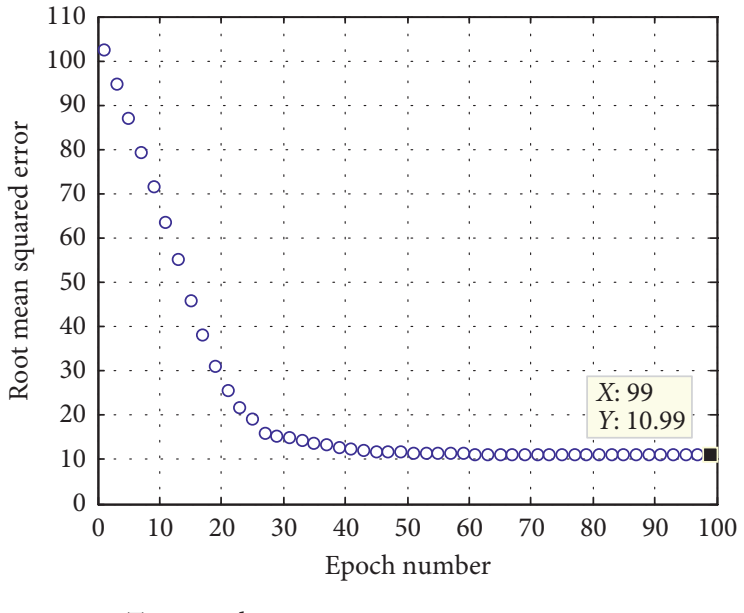

(b)

FIgURE 8: Error accumulation of the ANFIS model. (a) Training. (b) Testing.

TABLE 7: Performance results of the ANFIS model.

\begin{tabular}{lccc}
\hline & Training & Testing & Overall \\
\hline$R^{2}$ & 0.9624 & 0.9518 & 0.9584 \\
MSE & 87.9 & 119 & 89.5 \\
RMSE & 9.39 & 10.9 & 9.46 \\
$a_{20 \text {-index }}$ & 0.94 & 0.88 & 0.93 \\
Number of samples & 105 & 26 & 131 \\
\hline
\end{tabular}

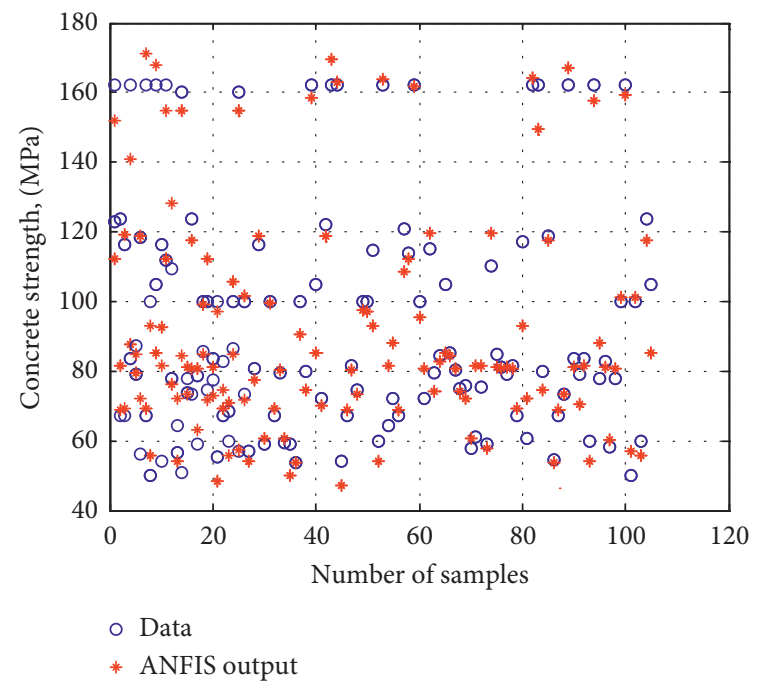

(a)

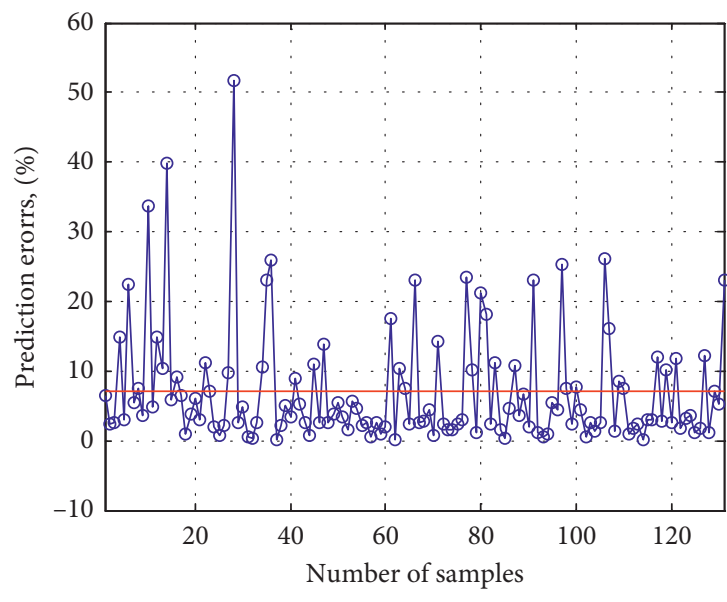

(b)

Figure 9: Performance of the ANFIS model. (a) Outputs versus inputs. (b) Absolute prediction errors.

strength of concrete. It is interesting to note that the amount of fiber had a minimal effect on the compressive strength of FRHSSCC. In other words, an increase in the amount of fiber from the minimum value found in this experimental dataset would have a limited effect on the 28-day compressive strength of FRHSSCC. 


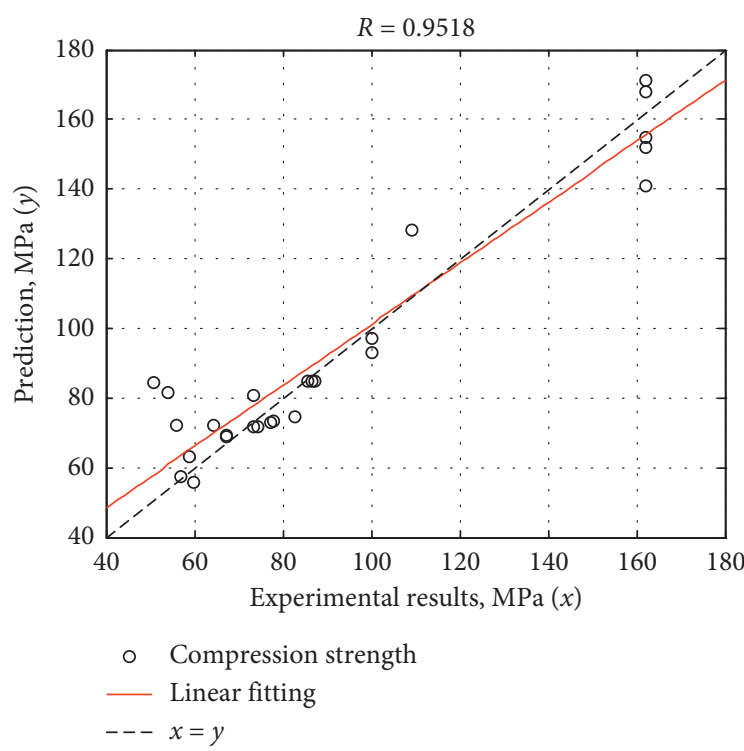

(a)

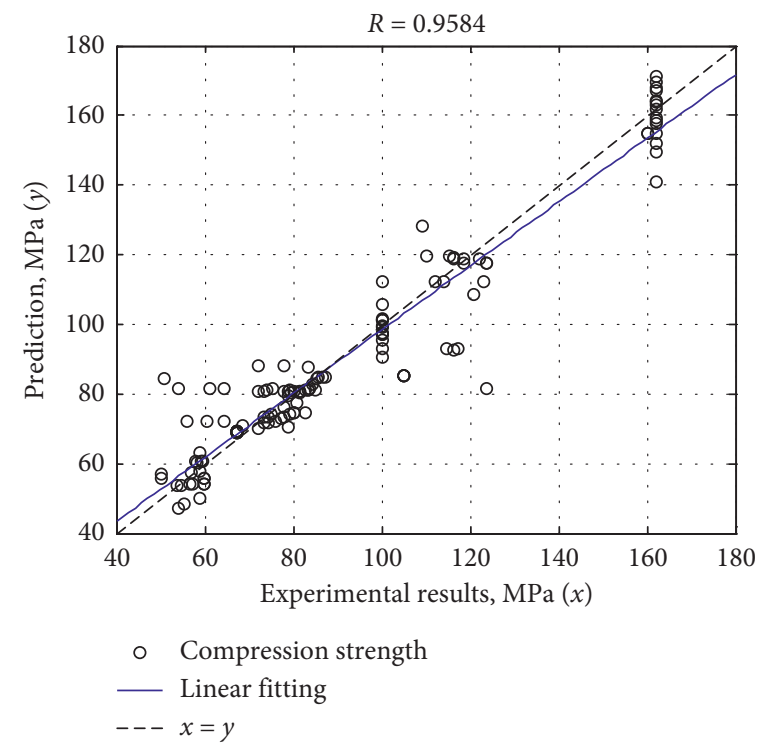

(b)

FIgURE 10: Linear regression plot of ANFIS performance. (a) Testing. (b) Overall.

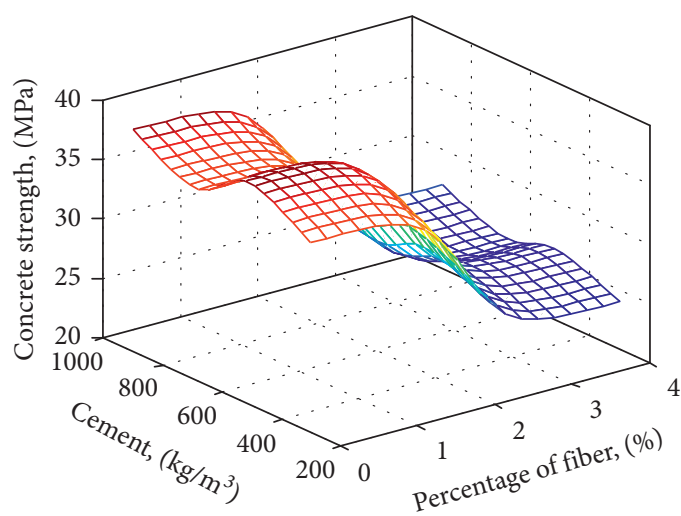

(a)

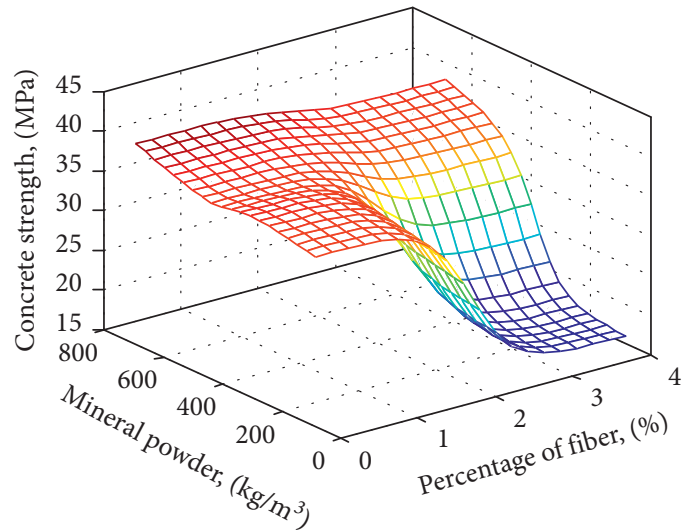

(c)

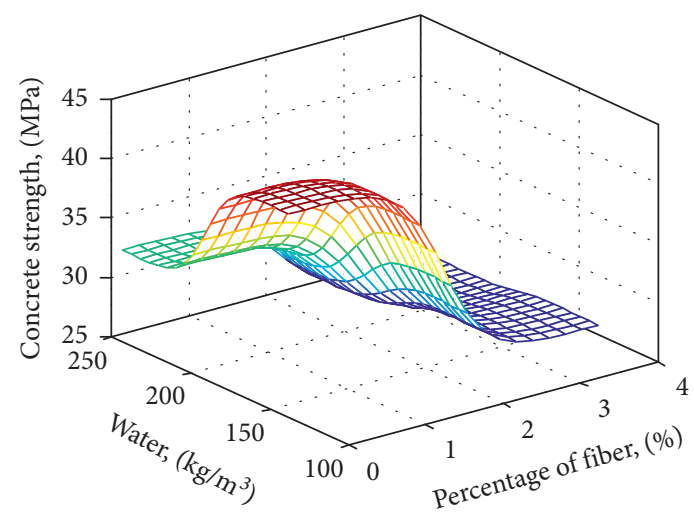

(b)

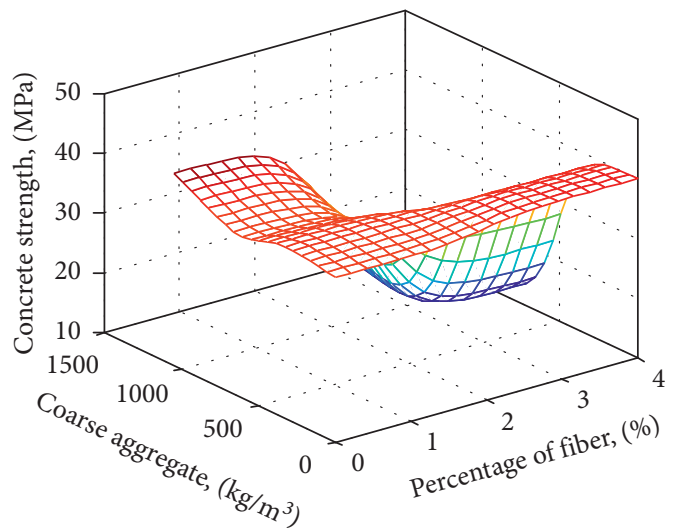

(d)

FIGURE 11: Relationship between different inputs and output. (a) VOF and CEM versus F28. (b) VOF and WTR versus F28. (c) VOF and MPR versus F28. (d) VOF and COA versus F28. 
TABLE 8: Input parameter data for sensitivity analysis.

\begin{tabular}{lccccc}
\hline Input parameters & LO & ML & MD & MH & HI \\
\hline FBT & 1 & 2.5 & 4 & 5.5 & 7 \\
VOF $(\%)$ & 0 & 1 & 2 & 3 & 4 \\
CEM $\left(\mathrm{kg} / \mathrm{m}^{3}\right)$ & 300 & 450 & 600 & 750 & 900 \\
WTR $\left(\mathrm{kg} / \mathrm{m}^{3}\right)$ & 138 & 164 & 191 & 217 & 243 \\
MPR $\left(\mathrm{kg} / \mathrm{m}^{3}\right)$ & 70 & 225 & 381 & 536 & 691 \\
WCR & 0.2 & 0.3 & 0.4 & 0.5 & 0.6 \\
FIA $\left(\mathrm{kg} / \mathrm{m}^{3}\right)$ & 451 & 647 & 842 & 1038 & 1233 \\
COA $\left(\mathrm{kg} / \mathrm{m}^{3}\right)$ & 0 & 261 & 521 & 782 & 1042 \\
\hline
\end{tabular}

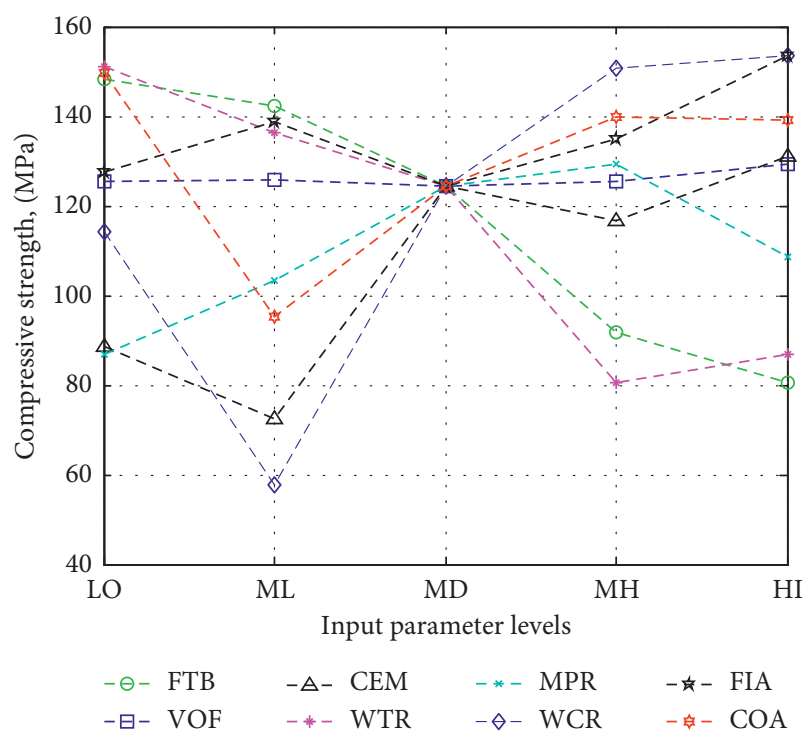

FIGURE 12: Compressive strength versus inputs.

\section{Conclusions}

In this research, the ANN and ANFIS models were developed to predict the 28-day compressive strength of fiberreinforced high-strength self-compacting concrete from 131 experimental mixtures. A series of ANN models were developed with different training algorithms and the number of neurons in the hidden layer to acquire the optimal model. The ANN model, with the Levenberg-Marquardt training algorithm and six nodes in the hidden layer, was found to work best with the experimental FRHSSCC data. In addition, both ANN and ANFIS models could predict the 28-day compressive strength at a high level of accuracy. In terms of statistical parameter, $R^{2}$, it is observed that the ANN model performed better than the ANFIS model.

The RMSE values of the ANN model for training, testing, and overall were $7.33 \mathrm{MPa}, 8.71 \mathrm{MPa}$, and $7.47 \mathrm{MPa}$, respectively. The absolute mean error between the actual FRHSSCC compressive strength and the predicted values was approximately six percent. With respect to the ANFIS model, the values of RMSE were 9.39 MPa for the training phase and 9.46 MPa for overall performance. These models can be employed as a reliable tool for selecting the optimized mix design for FRHSSCC. It is worth noting that the small number of test samples in the experimental input data is a limitation of this study. The performance of the proposed models would improve with an increase in the amount of input data. Finally, the sensitivity analysis results with the ANN model revealed that the 28-day compressive strength of FRHSSCC was more sensitive with the changes of water by cement ratio (WCR) parameter and less sensitive with varying amounts of fiber (VOF).

\section{Data Availability}

The data used to support the findings of this study are included in the article.

\section{Conflicts of Interest}

The authors declare no potential conflicts of interest with respect to the research, authorship, and/or publication of this article.

\section{Acknowledgments}

This research was funded by the National University of Civil Engineering (NUCE), Hanoi, Vietnam (Grant no. 29-2019/ KHXD-TĐ).

\section{References}

[1] A. Behnood, V. Behnood, M. Modiri Gharehveran, and K. E. Alyamac, "Prediction of the compressive strength of normal and high-performance concretes using M5P model tree algorithm," Construction and Building Materials, vol. 142, pp. 199-207, 2017.

[2] P. Dybel, "Effect of bond conditions on local bond-slip relationships of ribbed bars in high performance self-compacting concrete," Archives of Civil and Mechanical Engineering, vol. 19, no. 4, pp. 1399-1408, 2019.

[3] M. Ouchi, N. Sada-aki, T. Osterberg, S.-E. Hallberg, and M. Lwin, Applications of Self-Compacting Concrete in Japan, Europe and the United States, US Federal Highway Administration Office of Bridge Technology, Washingston, DC, USA, 2003.

[4] W. Zatar and T. Nguyen, "Mixture design study of fiberreinforced self-compacting concrete for prefabricated street light post structures," Advances in Civil Engineering, vol. 2020, Article ID 8852320, 7 pages, 2020.

[5] Z. Salari, B. Vakhshouri, and S. Nejadi, "Analytical review of the mix design of fiber reinforced high strength self-compacting concrete," Journal of Building Engineering, vol. 20, pp. 264-276, 2018.

[6] ACI, "Emerging technology series self-consolidating concrete," Reported by ACI Committee, vol. 237, 2007.

[7] T. T. Nguyen, T. N. Dao, S. Aaleti, K. Hossain, and K. J. Fridley, "Numerical model for creep behavior of axially loaded CLT panels," Journal of Structural Engineering, vol. 145, no. 1, Article ID 4018224, 2019.

[8] H. Long, "A sampling method for investigating self-healing property of concrete damaged by the drying shrinkage," Journal of Science and Technology in Civil Engineering, vol. 11, no. 6, pp. 97-103, 2017.

[9] T. T. Nguyen, T. N. Dao, S. Aaleti, J. W. van de Lindt, and K. J. Fridley, "Seismic assessment of a three-story wood building with an integrated CLT-lightframe system using RTHS," Engineering Structures, vol. 167, pp. 695-704, 2018. 
[10] T. T. Nguyen, Modeling of CLT Creep Behavior and Real-Time Hybrid Simulation of a CLT-LiFS Building, University of Alabama, Tuscaloosa, AL, USA, 2017.

[11] R. Ince, "Prediction of fracture parameters of concrete by artificial neural networks," Engineering Fracture Mechanics, vol. 71, no. 15, pp. 2143-2159, 2004.

[12] A. T. A. Dantas, M. Batista Leite, and K. De Jesus Nagahama, "Prediction of compressive strength of concrete containing construction and demolition waste using artificial neural networks," Construction and Building Materials, vol. 38, pp. 717-722, 2013.

[13] M. M. Alshihri, A. M. Azmy, and M. S. El-Bisy, "Neural networks for predicting compressive strength of structural light weight concrete," Construction and Building Materials, vol. 23, no. 6, pp. 2214-2219, 2009.

[14] T. T. Pham, T. T. Nguyen, L. N. Nguyen, and P. V. Nguyen, “A neural network approach for predicting hardened property of geopolymer concrete," International Journal of Geomate, vol. 19 , no. 74, pp. 176-184, 2020.

[15] H. Naderpour, A. H. Rafiean, and P. Fakharian, "Compressive strength prediction of environmentally friendly concrete using artificial neural networks," Journal of Building Engineering, vol. 16, pp. 213-219, 2018.

[16] A. Öztaş, M. Pala, E. Özbay, E. Kanca, N. Çağlar, and M. A. Bhatti, "Predicting the compressive strength and slump of high strength concrete using neural network," Construction and Building Materials, vol. 20, no. 9, pp. 769-775, 2006.

[17] J. Sobhani, M. Najimi, A. R. Pourkhorshidi, and T. Parhizkar, "Prediction of the compressive strength of no-slump concrete: a comparative study of regression, neural network and anfis models," Construction and Building Materials, vol. 24, no. 5, pp. 709-718, 2010.

[18] R. Siddique, P. Aggarwal, and Y. Aggarwal, "Prediction of compressive strength of self-compacting concrete containing bottom ash using artificial neural networks," Advances in Engineering Software, vol. 42, no. 10, pp. 780-786, 2011.

[19] F. Khademi, M. Akbari, S. M. Jamal, and M. Nikoo, "Multiple linear regression, artificial neural network, and fuzzy logic prediction of 28 days compressive strength of concrete," Frontiers of Structural and Civil Engineering, vol. 11, no. 1, pp. 90-99, 2017.

[20] T. T. Nguyen and K. Dinh, "An artificial intelligence approach for concrete hardened property estimation," Journal of Science and Technology in Civil Engineering, vol. 14, no. 2, pp. 40-52, 2020.

[21] J. Sobhani and M. Najimi, "Numerical study on the feasibility of dynamic evolving neural-fuzzy inference system for approximation of compressive strength of dry-cast concrete," Applied Soft Computing, vol. 24, pp. 572-584, 2014.

[22] K. Prasad, A. K. Gorai, and P. Goyal, "Development of ANFIS models for air quality forecasting and input optimization for reducing the computational cost and time," Atmospheric Environment, vol. 128, pp. 246-262, 2016.

[23] T. T. Nguyen and K. Dinh, "Prediction of bridge deck condition rating based on artificial neural networks," Journal of Science and Technology in Civil Engineering, vol. 13, no. 3, pp. 15-25, 2019.

[24] E. Hong, A. M. Yeneneh, T. K. Sen, H. M. Ang, and A. Kayaalp, "ANFIS based modelling of dewatering performance and polymer dose optimization in a wastewater treatment plant," Journal of Environmental Chemical Engineering, vol. 6, no. 2, pp. 1957-1968, 2018.

[25] D. J. Armaghani, G. D. Hatzigeorgiou, C. Karamani, A. Skentou, I. Zoumpoulaki, and P. G. Asteris, "Soft computing-based techniques for concrete beams shear strength," Procedia Structural Integrity, vol. 17, pp. 924-933, 2019.

[26] P. Sarir, J. Chen, P. G. Asteris, D. J. Armaghani, and M. M. Tahir, "Developing GEP tree-based, neuro-swarm, and whale optimization models for evaluation of bearing capacity of concrete-filled steel tube columns," Engineering with Computers, 2019.

[27] H. Jing, H. Nikafshan Rad, M. Hasanipanah, D. Jahed Armaghani, and S. N. Qasem, "Design and implementation of a new tuned hybrid intelligent model to predict the uniaxial compressive strength of the rock using SFS-ANFIS," Engineering with Computers, 2020.

[28] D. J. Armaghani, P. G. Asteris, S. A. Fatemi et al., "On the use of neuro-swarm system to forecast the pile settlement," Applied Sciences, vol. 10, no. 6, 2020.

[29] M. Nikbin, R. S. Rahimi, and H. Allahyari, "A new empirical formula for prediction of fracture energy of concrete based on the artificial neural network," Engineering Fracture Mechanics, vol. 186, pp. 466-482, 2017. 